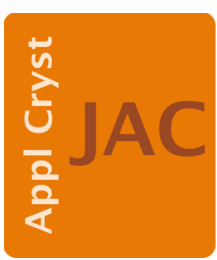

JOURNAL OF

APPLIED

CRYSTALLOGRAPHY

Volume 51 (2018)

Supporting information for article:

Operando powder X-ray diffraction study of P2-NaXNi0.3Mn0.7O2 cathode material during electrochemical cycling

Solveig Kjeldgaard, Steinar Birgisson, Anette Gert Kielland and Bo Brummerstedt Iversen 


\section{S1. Experimental}

\section{S1.1. Stagnated structure}

During charging (in first and second cycle) a voltage plateau is observed at 3.8-4.0 V ( 4.0 V plateau). The $\sim 4.0 \mathrm{~V}$ plateau is considerably shorter in the second cycle, indicating that the redox reaction happening there is irreversible. The $\sim 4.0 \mathrm{~V}$ plateau coincides with a stagnation of all structural parameters of P2. As observed in Fig. 3 and 4, the sodium loading and unit cell parameters are constant in this region. Furthermore, the scale factor of P2-NNMO (normalized to the scale factor of aluminium (current collector)) is constant in this region, see Figure S1. Together, this indicates that P2-NNMO is electrochemically inactive in this voltage range. The capacity extracted during the voltage plateau therefore stems from a separate redox process, not including P2-NNMO. This observation clearly demonstrates that sodium ion extraction/insertion from crystalline electrode materials cannot in all cases be directly estimated from capacity measurements. The same probably holds true for lithium ion battery materials.

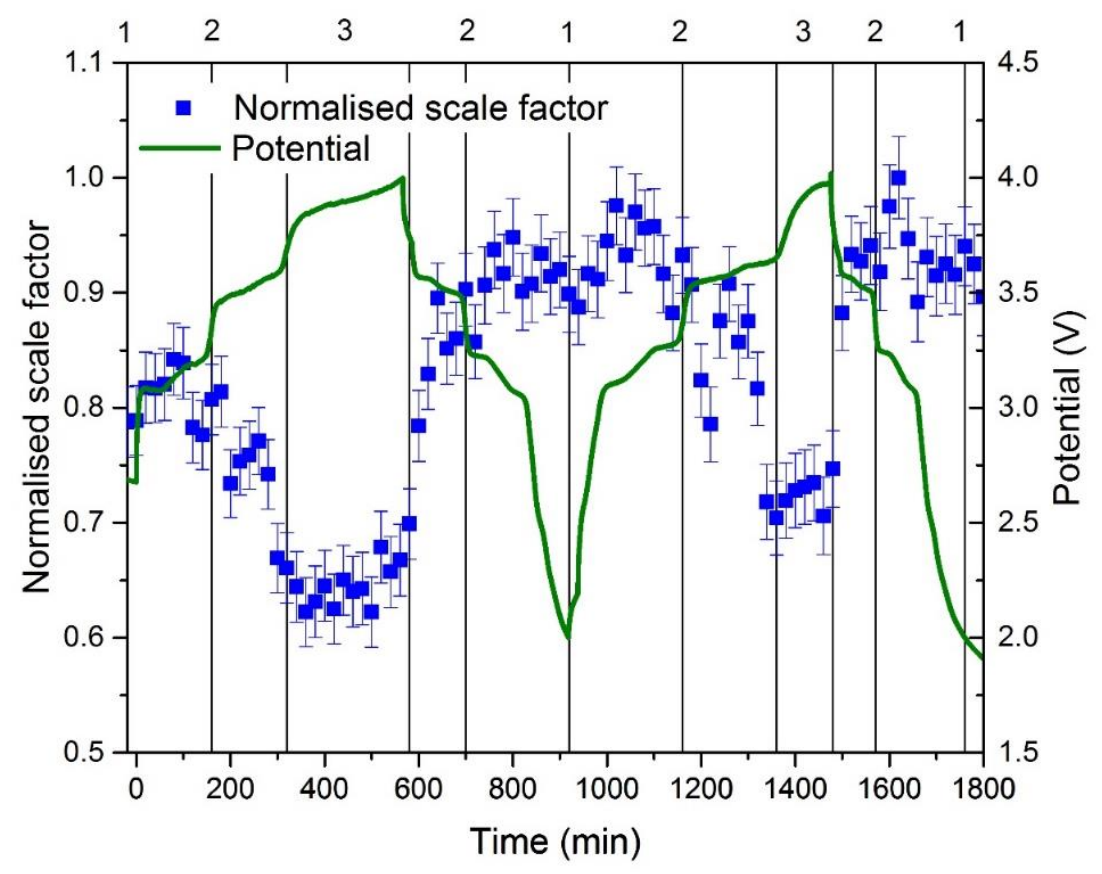

Figure S1 Normalized scale factor and voltage curve. The normalized scale factor is constant during the voltage plateau at 3.8-4.0 V, indicating that $\mathrm{P} 2-\mathrm{NNMO}$ is inactive in this voltage range. 
The redox process happening in the $\sim 4.0 \mathrm{~V}$ plateau might originate from either electrolyte decomposition at high voltage, extraction of sodium from an amorphous phase contained in the NNMO material or be an artefact of the special design of the operando cell. Note that it is likely that the NNMO material contains sodium rich amorphous phase since the sodium to transition metal ratio introduced in the high temperature calcination was $\mathrm{x} \approx 1.0$ even though the $\mathrm{P} 2$-NNMO phase only contained $\mathrm{x} \approx 0.62$ after the synthesis. To determine the origin of the $\sim 4.0 \mathrm{~V}$ plateau the NNMO material was cycled between $4.5-2.0 \mathrm{~V}$ at $0.04 \mathrm{~mA}$ in a coin cell type sodium half-cell, see capacity-voltage (CV) curve Figure S2a. Upon first charge the $\sim 4.0 \mathrm{~V}$ plateau appears before the onset of a long plateau at $\sim 4.2 \mathrm{~V}$. This shows that the $\sim 4.0 \mathrm{~V}$ plateau is not an artefact of the operando cell and must originate from the NNMO material or the electrolyte. The $\sim 4.0 \mathrm{~V}$ plateau disappears upon further cycling showing unambiguously that the process is irreversible. The CV curve of NNMO was further compared to a coin cell made in an identical manner but containing a material where the sodium to transition metal ratio during the calcination was $\mathrm{x} \approx 0.7$ (NNMO-0.7). The crystalline phase of NNMO-0.7 was found to be $\mathrm{P} 2$ with $\mathrm{x} \approx 0.60$, see below, and will therefore contain considerably lower amount of amorphous phases compared to NNMO. As seen in Figure S2b the NNMO-0.7 material does not produce a $\sim 4.0 \mathrm{~V}$ plateau showing that the $\sim 4.0 \mathrm{~V}$ plateau of the NNMO material most probably originates from an electrochemical reaction of a sodium rich amorphous phase contained in the material. Similar sodium rich amorphous phase has been shown to be present in $\mathrm{P} 2$ type $\mathrm{Na}_{\mathrm{x}} \mathrm{Co}_{0.7} \mathrm{Mn}_{0.3} \mathrm{O}_{2}$ synthesized with $\mathrm{x} \approx$ 1.0 using a similar methodology (Birgisson et al., 2017).

The redox reaction of the amorphous phase in the $\sim 4.0 \mathrm{~V}$ plateau is not considered further, as the focus is on the crystalline $\mathrm{P} 2$ phase in the NNMO cathode material.
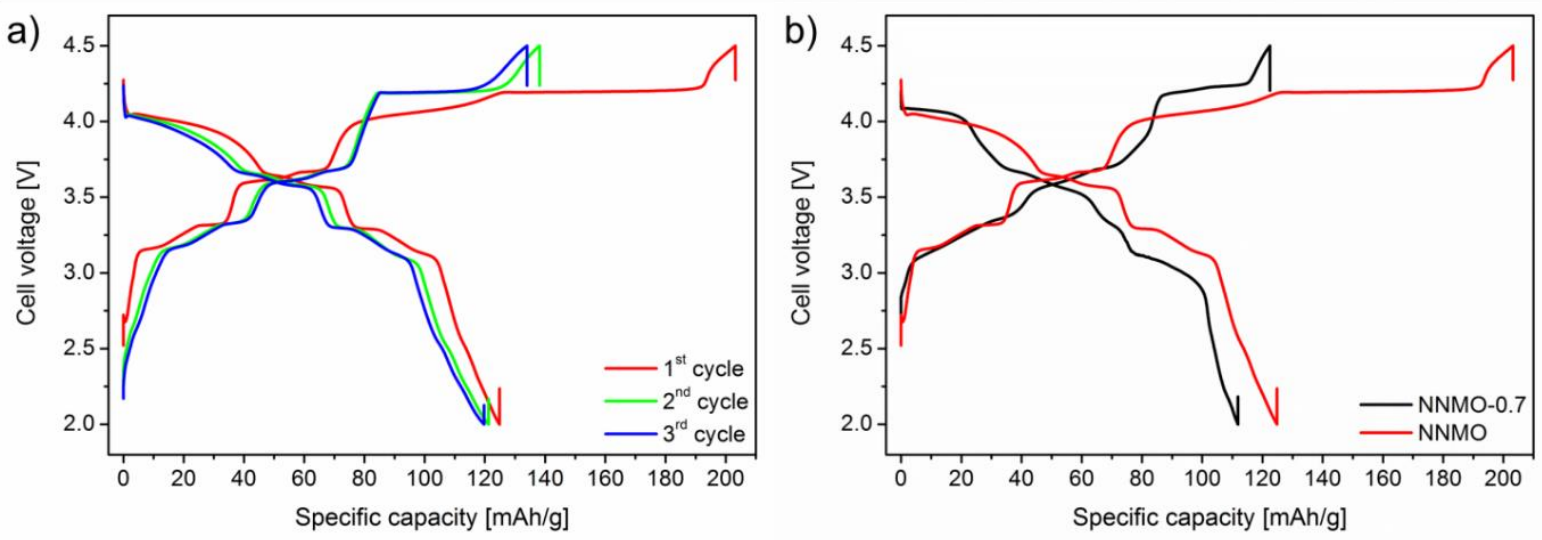

Figure S2 a) Cycling of NNMO between 4.5-2.0 V at 0.04 mA. b) Comparison of the first cycle of NNMO and NNMO-0.7 cycled between 4.5-2.0 V at 0.04 mA. 


\section{S1.2. Structure of as-prepared NNMO-0.7 material}

PXRD data of the as prepared NNMO-0.7 material is fitted to the P2 structure as was done for NNMO, see Figure S3 and Tables S1-S2.

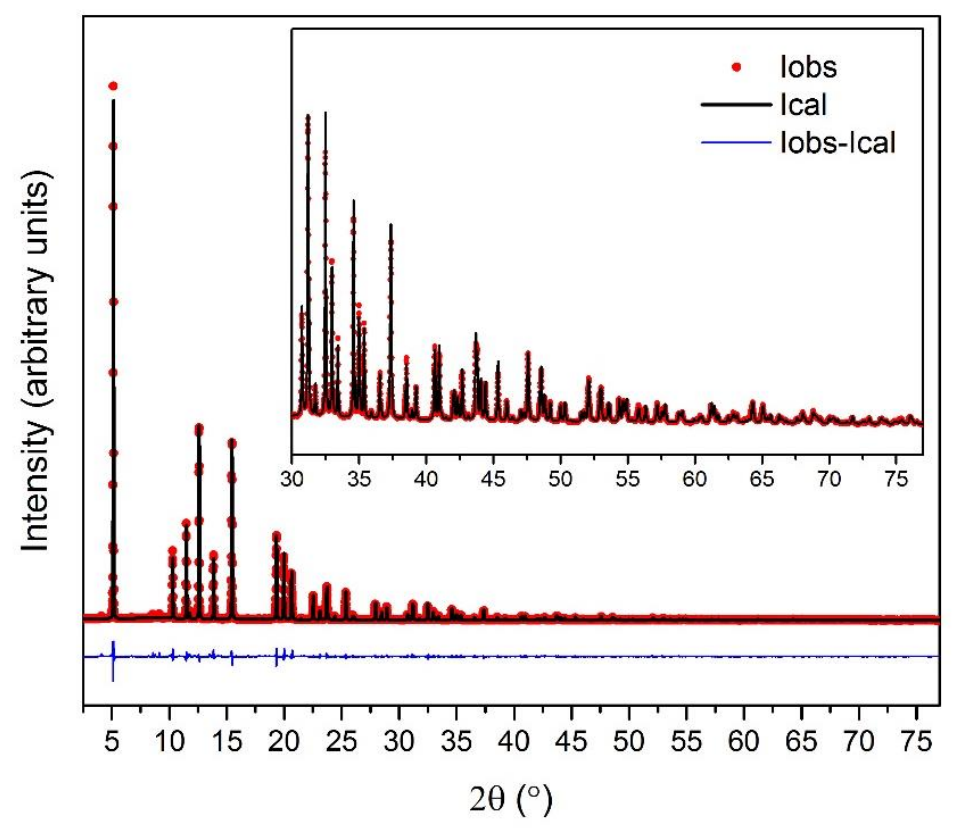

Figure S3 Rietveld refinement fit of the as prepared P2-NNMO-0.7

Table S1 Crystallographic details for the as prepared P2-NNMO-0.7

\begin{tabular}{ll}
\hline Space group & $\mathrm{P}_{3} / \mathrm{mmc}$ \\
\# data points & 7797 \\
\# background points & 14 \\
\# fitted parameters & 29 \\
$\mathrm{R}_{\text {Bragg }}$ & 3.51 \\
$\mathrm{R}_{\mathrm{F}}$ & 3.88 \\
$\mathrm{R}_{\mathrm{wp}}$ & 7.62 \\
$\mathrm{R}_{\text {exp }}$ & 1.95 \\
Lattice parameter a/b $[\AA]$ & $2.88501(1)$ \\
Lattice parameter c $[\AA]$ & $11.14283(7)$ \\
\hline
\end{tabular}


Table S2 Refined atomic parameters for the as prepared P2-NNMO-0.7

\begin{tabular}{llllll}
\hline Site & $\mathbf{X}$ & $\mathbf{Y}$ & $\mathbf{Z}$ & Occupancy & \multicolumn{1}{c}{ Biso } \\
$\mathrm{Na}_{\mathrm{e}}$ & $0.277(3)$ & $0.555(7)$ & 0.75 & $0.39(1)$ & $0.98(19)$ \\
$\mathrm{Na}_{\mathrm{f}}$ & 0 & 0 & 0.25 & $0.21(1)$ & $1.8(2)$ \\
$\mathrm{Ni} / \mathrm{Mn}$ & 0 & 0 & 0.5 & $0.30 / 0.70$ (fixed) & $0.424(7)$ \\
$\mathrm{O}$ & 0.33333 & 0.66667 & $0.0908(2)$ & 2 (fixed) & $0.65(3)$ \\
\hline
\end{tabular}

\section{S2. Rietveld refinement of operando data}

Two phases are included in the refinement; P2-NNMO and aluminium (current collector). Additional peaks arising from the current collector that cannot be fit to the aluminium phase are present. These are marked with green triangles in figure S4.

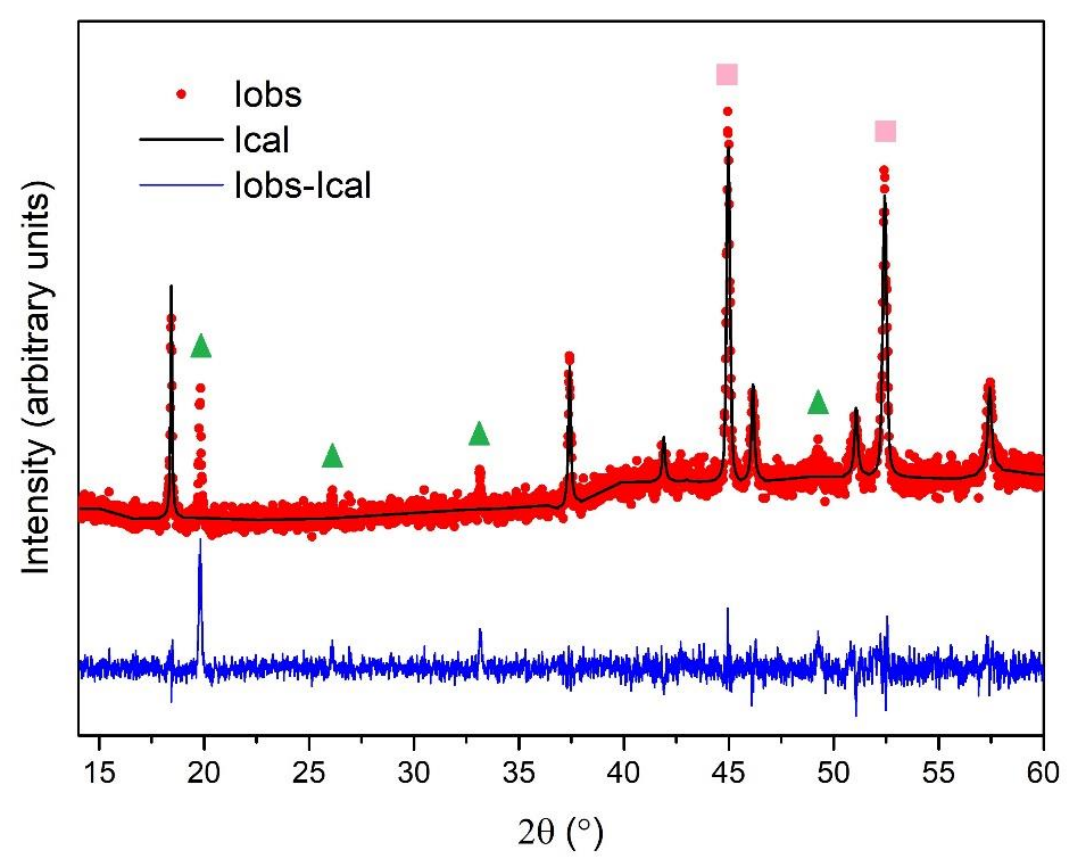

Figure S4 Rietveld refinement fit of first frame of sequential fitting. Pink squares indicate the Aluminium phase. Green triangles indicate peaks that arise from the current collector but that cannot be fit to the aluminium phase. 
Table S3 Crystallographic details for the first frame

\begin{tabular}{ll}
\hline \# data points & 3021 \\
\# background points & 17 \\
\# fitted parameters & 31 \\
\hline
\end{tabular}

Table S4 Crystallographic details for the first frame

\begin{tabular}{lll}
\hline Phase & P2-NNMO & Aluminum \\
Space group & $\mathrm{P} 6_{3} / \mathrm{mmc}$ & $\mathrm{Fm} \overline{3} \mathrm{~m}$ \\
$\mathrm{R}_{\mathrm{Bragg}}$ & 13.6 & 5.14 \\
$\mathrm{R}_{\mathrm{F}}$ & 8.51 & 2.51 \\
Lattice parameter $a / b[\AA]$ & $2.887(3)$ & $4.048(3)$ \\
Lattice parameter $c[\AA]$ & $11.15(2)$ & - \\
\hline
\end{tabular}

